\title{
A SINGLE-BLOCK TRL TEST FIXTURE FOR THE CRYOGENIC CHARACTERIZATION OF PLANAR MICROWAVE COMPONENTS
}

\author{
M. Mejía ${ }^{{ }^{*}}$, A. S. Creason ${ }^{1 *}$, S. S. Toncich ${ }^{2}$, B. T. Ebihara ${ }^{1}$, \\ and F. A. Miranda \\ ${ }^{1}$ National Aeronautics and Space Administration \\ I.ewis Research Center, Cleveland, OH 44135. USA \\ 2 Bird Electronics \\ Solon. OH 44139, USA
}

\begin{abstract}
The High-Temperature-Superconductivity (HTS) group of the RF Technology Branch. Space Electronics Division, is actively involved in the fabrication and cryogenic characterization of planar microwave components for space applications. This process requires fast, reliable, and accurate measurement techniques not readily available. A new calibration standard/test fixture that enhances the integrity and reliability of the component characterization process has been developed. The fixture consists of $50 \Omega$ thru, reflect, delay, and device under test gold lines etched onto a $254 \mu \mathrm{m}\left(0.010^{\prime \prime}\right)$ thick alumina substrate. The Thru-Reflect-Line (TRL) fixture was tested at room temperature using a $30 \Omega, 7.62 \mathrm{~mm}$ ( $300 \mathrm{mil})$ long, gold line as a known standard. Good agreement between the experimental data and the data modelled using Sonnet's $\mathrm{em}^{\mathrm{c}}$ software was obtained for both the return $\left(S_{11}\right)$ and insertion $\left(S_{21}\right)$ losses. A gold two-pole bandpass filter with a $7.3 \mathrm{GHz}$ center frequency was used as our device under test (DUT), and the results compared with those obtained using a Short-Open-Load-Thru (SOLT) calibration technique. Minimum insertion losses of $3.85 \mathrm{~dB}$ and $5.41 \mathrm{~dB}$ were measured with the TRL and SOLT calibration techniques, respectively. Room temperature retum losses of $25.56 \mathrm{~dB}$ and $16.86 \mathrm{~dB}$ were measured using the TRL and SOLT calibration techniques, respectively. When used to perform calibrations and testing at cryogenic temperatures, the TRL fixture exhibited insertion and return loss data superior to its SOLT counterpart. A detailed description of the cryogenic components of the TRL fixture as well as results of their cryogenic characterization are presented.
\end{abstract}

FJPPNASA student intern; University of Pennsylvania, Philadelphia PA 19104 USA.

“OAINASA student intern; Ohio Northern University, Ada OH 45810 USA. 


\section{INTRODUCTION}

Microwave systems for space applications, such as front end receivers, demand low noise, low loss components with stringent frequency response properties. Therefore, the individual microwave components that make up a particular system should be thoroughly characterized, so that their intrinsic performance (i.e., excluding the effects of the matching network) can be evaluated. It has been shown that the performance of semiconductor-based microwave components improves at cryogenic temperatures'. Also, it has been demonstrated that at cryogenic temperatures, High-Temperature-Superconductors (HTS) have greatly reduced conductor losses in microwave components with respect to those fabricated using nomal metal conductors such as gold and copper'. Therefore, the overall operation of microwave systems can be improved by operating at cryogenic temperatures (e.g., $77 \mathrm{~K}$ ), and by optimizing individual component performance at those temperatures. In this paper. we describe the design. fabrication. and testing process of a new Thru-Reflect-Line (TRL) calibration method that will allow for the fast and accurate characterization of planar microwave components at cryogenic temperatures. Results on the performance of this fixture at room and cryogenic temperatures will be presented.

\section{EXPERIMENTAL APPROACH}

An Automatic Network Analyzer (ANA) is commonly used to characterize microwave components. The ANA. however. must be calibrated to establish reference planes before any measurements can be taken. These reference planes indicate how far along the system the calibration will take into account measuring network and test-fixture losses (e.g., losses due to coaxial cables, coaxial-to-microstrip transitions, wire bonding connections. ctc.), so that the losses of the device-under-test (DUT) can be determined more accurately. Often used in the characterization of microwave components, the TRL calibration establishes the reference planes beyond the electrical launchers, accounting for losses due to the coaxial-to-microstrip transition. The TRL calibration technique is typically implemented in the "split-block" configuration shown in Figure 1. While this calibration works well at a room temperature, its split-block configuration proves cumbersome for measurements at cryogenic temperatures and under vacuum conditions. lor example, each calibration step would require a different block to be wire bonded, placed under vacuum, cooled to the desired cryogenic temperatures, recorded for measurements, and returned to room temperature and pressure conditions ${ }^{3}$. Due to the cycling. multiple wiring, and possible drifting of the ANA reference levels, such a process would be time consuming and more prone to error. To improve the calibration process at cryogenic temperatures, the following experimental approach was initiated:

- Develop a TRL, calibration standard and test fixture that would:

1. Fliminate repeated temperature cycling and vacuum disruption (speed).

2. Allow for electromagnetic coupling repeatability (accuracy).

3. Have a simple and inexpensive implementation (affordability).

- Optimize TR1, calibration at room temperature.

- Optimize TRL calibration at cryogenic temperatures.

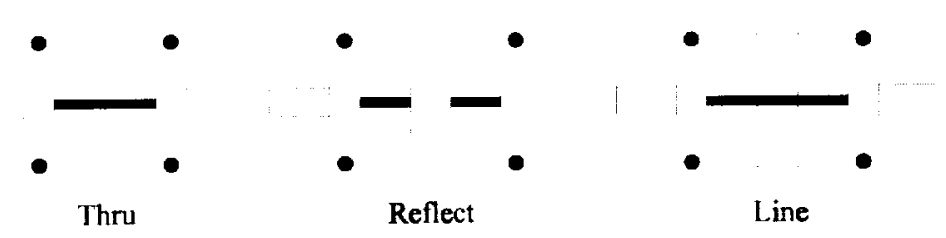

Figure 1. TRL "split-block" configuration. 


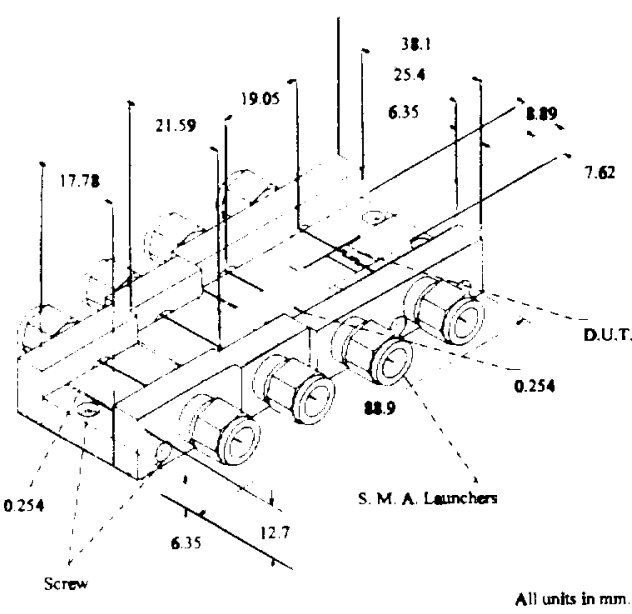

Figure 2. "Single-block" TRL. schematic.

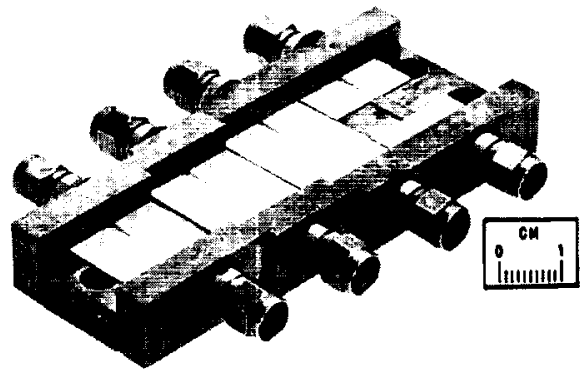

Figure 3. TRL test fixture.

The above approach led to a single-block TRL design. A schematic representation and the actual fixture are shown in Figures 2 and 3, respectively. The fixture consists of $254 \mu \mathrm{m}\left(0.010^{\prime \prime}\right)$ wide thru, reflect, delay and DUT gold lines. The $50 \Omega$ input/output lines were patterned on a 254 $\mu \mathrm{m}\left(0.010^{\prime \prime}\right)$ thick alumina substrate $\left(88.9 \times 38.1 \mathrm{~mm}\left(3.5^{\prime \prime} \times 1.0^{\prime \prime}\right)\right)$. A brass block supports the SMA launchers used for the coaxial-to-microstrip transition. Each microstrip line was split and mounted at $19.05 \mathrm{~mm}\left(0.75^{\prime \prime}\right)$ intervals onto the brass piece using silver-based conductive epoxy. For the measurements, modifications corresponding to our selected standards were introduced into the TRL calibration routine of the HP-8510C ANA 4 .

For the cryogenic testing. a custom made vacuum chamber was fabricated. The aluminum chamber was designed so that it can be mounted on the second stage of a closed-cycle helium gas refrigerator. It also features feed-throughs to accommodate the coaxial cables necessary to couple the microwave signal from the ANA with the TRL, test fixture. Figures $4-6$ show the interior and exterior of the chamber and the experimental set up for cryogenic testing. The $3.5 \mathrm{~mm}$ coaxial cables used to couple the TRL test fixture with the microwave signal originating in the ANA were custom made and consisted of a silver plated beryllium copper inner conductor, a Teflon (PTFE) dielectric, and a stainless steel outer conductor as shown in Figure 7. Fifty-six cables were fabricated to meet the electrical specifications of a return loss $\left(\mathrm{S}_{11}\right)$ better than $18.25 \mathrm{~dB}$ and an insertion loss $\left(\mathrm{S}_{21}\right)$ less than 0.7 $\mathrm{dB}$ in the $0.0-10.0 \mathrm{GHz}$ frequency range. The test fixture was mounted inside the vacuum chamber,

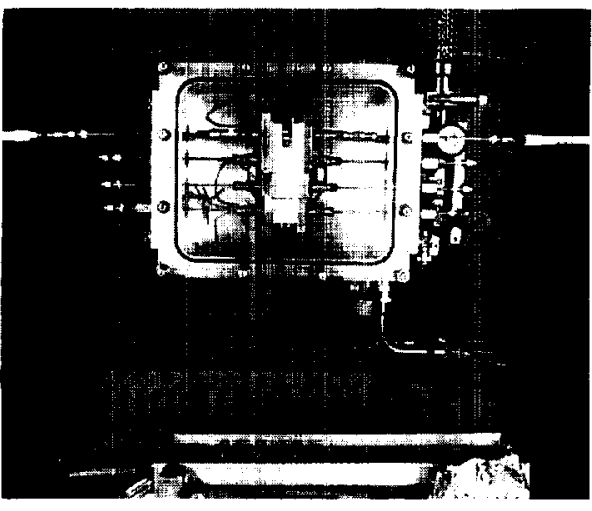

Figure 4. TRL mounted in testing configuration.

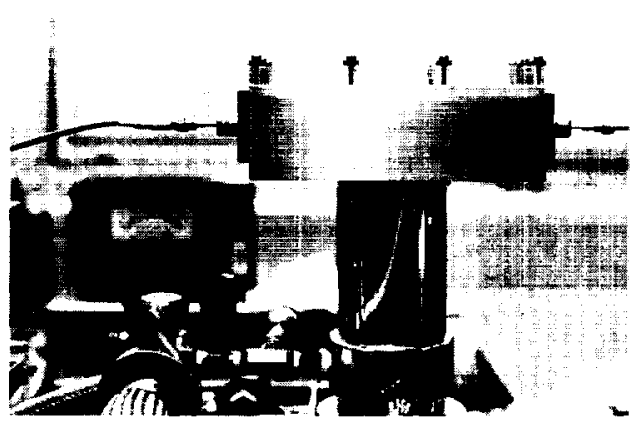

Figure 5. Vacuum chamber and refrigerator. 


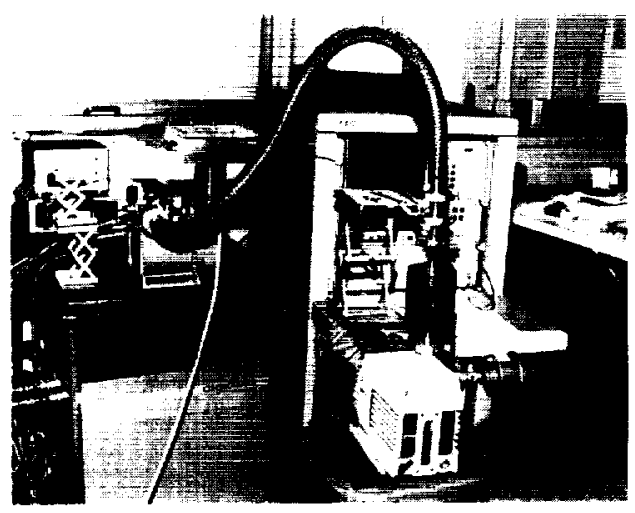

Figure 6. TRL testing set up.

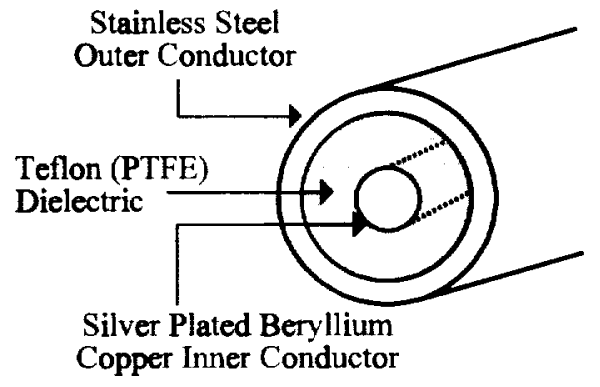

Figure 7. Custom made coaxial cable.

which was then tested to see if it could maintain vacuum conditions both at room and at cryogenic temperatures. A vacuum quality epoxy (Torr Seal $\mathbb{R}$ ) was applied to the joint of the coaxial cable and SMA connectors to protect against possible vacuum leakage.

\section{RESULTS}

The single-block TRL test fixture was tested at room temperature by using a $30 \Omega, 7.62 \mathrm{~mm}$ ( 300 mil) long, gold line as the DUT. $S_{11}$ and $S_{21}$ for this line were measured, and the experimental data compared with the modelled data for the line using Sonnet's $\boldsymbol{e m}^{\mathrm{C}}$ softwares. The experimental and the
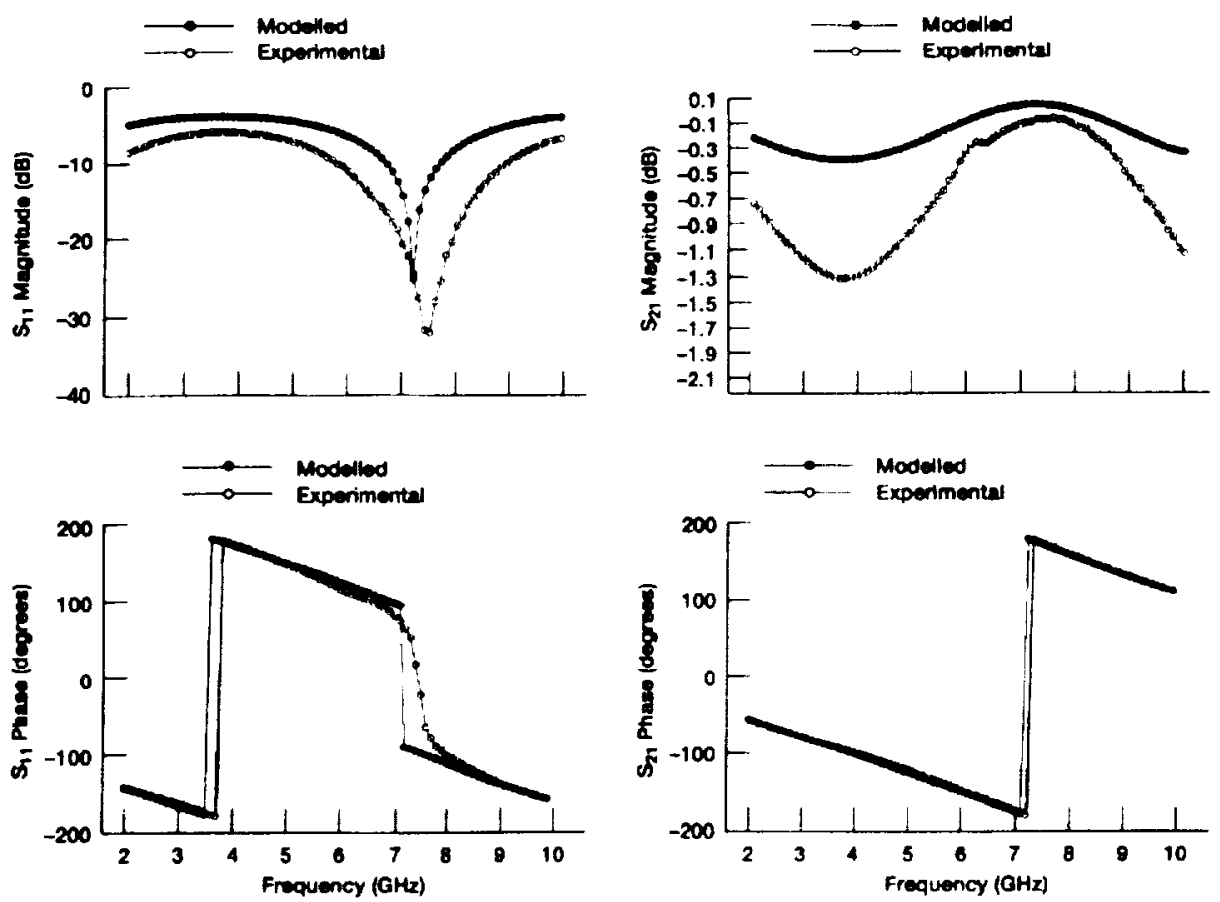

Figure 8. Insertion loss $\left(S_{21}\right)$ and return loss $\left(S_{11}\right)$ vs. frequency for a $30 \Omega, 7.62 \mathrm{~mm}(300 \mathrm{mil})$ long, gold line on $254 \mu \mathrm{m}$ ( $10 \mathrm{mil}$ ) thick alumina at $298 \mathrm{~K}$. 

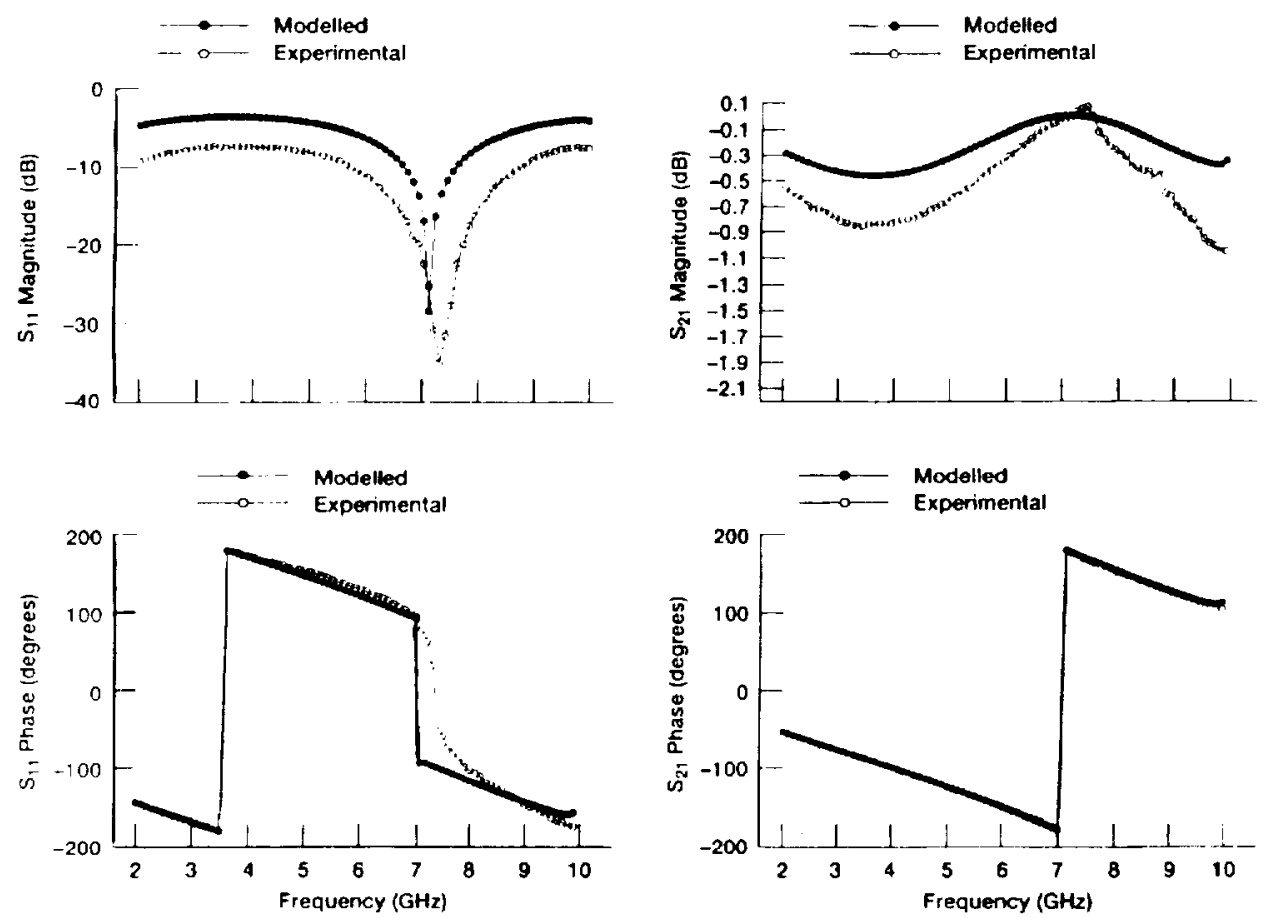

Figure 9. Insertion loss $\left(S_{21}\right)$ and return loss $\left(S_{11}\right)$ vs. frequency for a $30 \Omega, 7.62 \mathrm{~mm}$ (300 mil) long, gold line on $508 \mu \mathrm{m}(20 \mathrm{mil})$ thick alumina at $298 \mathrm{~K}$.
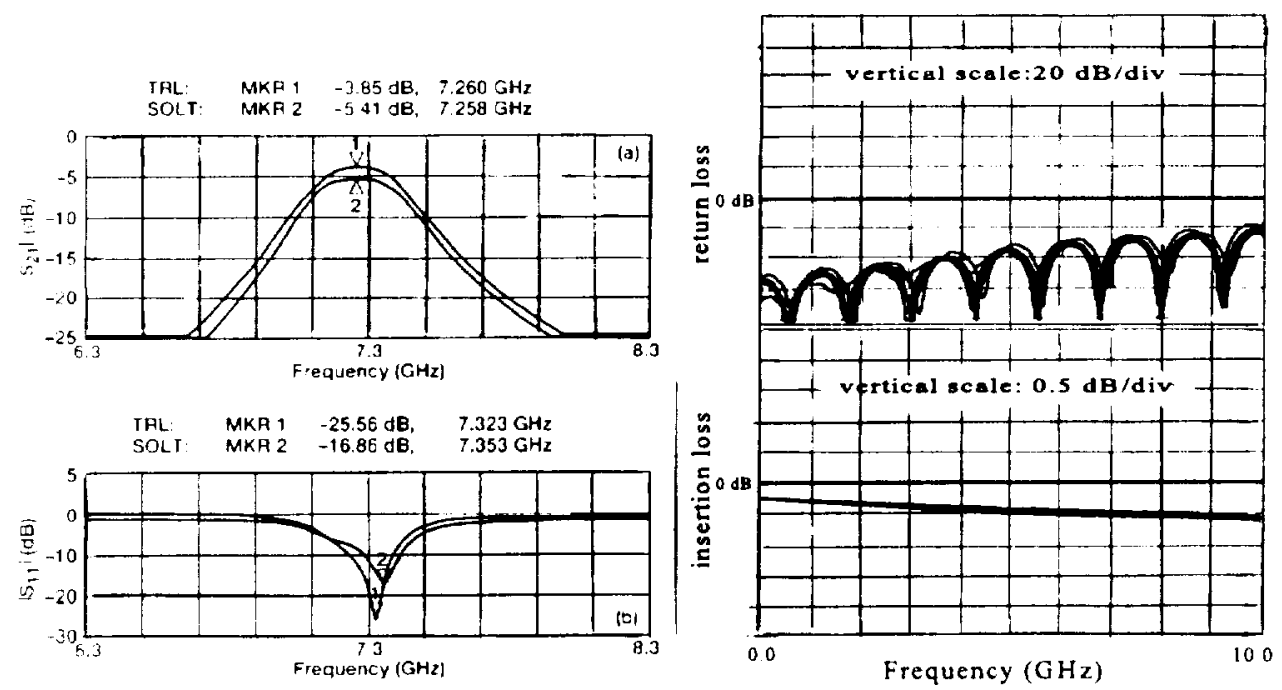

Figure 10. Insertion $\left(S_{3}\right)$ and return $\left(S_{11}\right)$ losses vs fiequency for a gold two-pole bandpass filter with a $7.3 \mathrm{GH} z$ center frequency. Both calibrations were performed at room temperature and outside the vacuum chamber.

Figure 11. Insertion $\left(S_{21}\right)$ and return $\left(S_{11}\right)$ for coixial cables. 
Table 1. Pressure and temperature data for experimental set up

\begin{tabular}{|c|c|c|c|}
\hline Time & Sensor A* & Sensor B** & Pressure \\
\hline $1-0$ & $298 \mathrm{~K}$ & $296 \mathrm{~K}$ & ATM \\
\hline $1-40$ minutes $* * *$ & $298 \mathrm{~K}$ & $296 \mathrm{~K}$ & 4.0 Pa (30 millitorrs) \\
\hline $1-3$ hours & $58 \mathrm{k}$ & $32 \mathrm{~K}$ & $0.133 \mathrm{~Pa}(\% 1$ millitorr $)$ \\
\hline
\end{tabular}

* mounted on test fixture

** mounted underneath of refrigerator cold linger

**** refrigerator turned on

modelled data for the magnitude and phase of $S_{11}$ and $S_{21}$ are shown in Figures 8 and 9 . Observe that good agreement exists between the experimental and modelled data. not only for the calibration circuit on the $254 \mu \mathrm{m}$ ( $10 \mathrm{mil})$ thick alumina substrate. but also for a similar circuit on a $508 \mu \mathrm{m}$ ( $20 \mathrm{mil}$ ) thick alumina substrate. The slight discrepancies between the experimental and modelled data could be due to uncertainties in the line's dimensions and unaccounted for radiation losses.

The repeatability of the TRI calibration standard was tested by reassembling the thru standard immediately after calibration. This resulted in a return loss better than $39 \mathrm{~dB}$ and an insertion loss of less than $0.02 \mathrm{~dB}$ from 0.0 to $10.0 \mathrm{GHz}$.

The next step in the verification of the TRL fixture was to compare its performance at room temperature with that of a standard Short-Open-Load-Thru (SOI T) calibration. a method that is frequently used in the characterization of microwave components. For this test, a gold two-pole bandpass filter, designed to exhibit a $7.3 \mathrm{GH}$ z center frequency. was used as a DU $\mathrm{T}^{2}$. The test fixture was calibrated outside the vacuum chamber using both the SOLT and TRL methods and the filter was tested in each case. As shown in Figure 10, the quality of data measured using the TRL. calibration surpasses that of its SOL T counterpart, showing improvement on insertion losses near $1.5 \mathrm{~dB}$ and on return losses near $10 \mathrm{~dB}$ at the center frequency. The observed deviations between the designed and experimental center frequencies are most prohably due to filter fabrication issues ${ }^{2}$.

In preparation for testing the fixture at cryogenic temperatures, each coaxial cable was tested to determine its return and insertion losses. Typical results for a set of eight cables are shown in Figure 11. Note that the cable to-cable signal propagation properties are very similar and satisfy the aforementioned specifications for return and insertion losses. Afterwards, the cables were assembled in the vacuum chamber (see Figures 4 and 5) and the TRI. fixture was mounted as described earlier. The whole system was then tested to determine the quality of the vacuum and cooling properties. Complete the TRL chamber held satisfactory vacuum conditions and reached acceptable cryogenic temperatures (see Table 1).

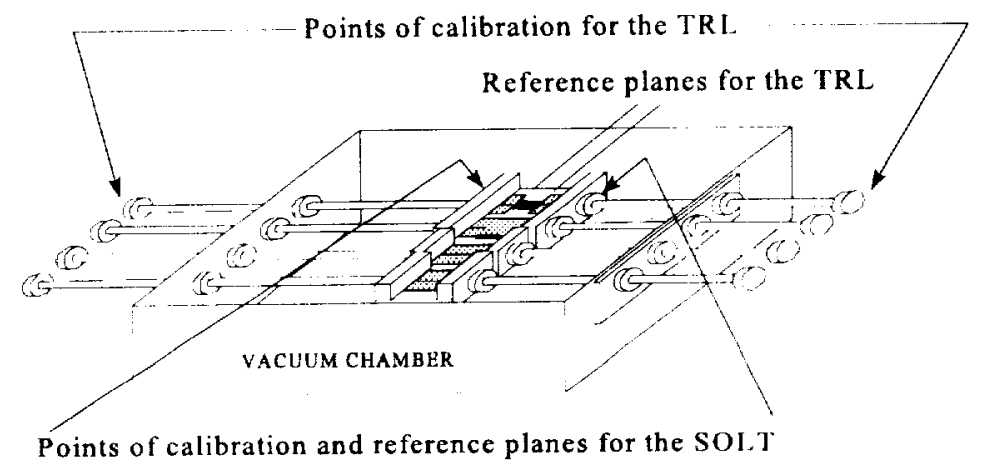

Figure 12. Points of calibration and established reference planes for the TRL and SOLT calibration techniques. 

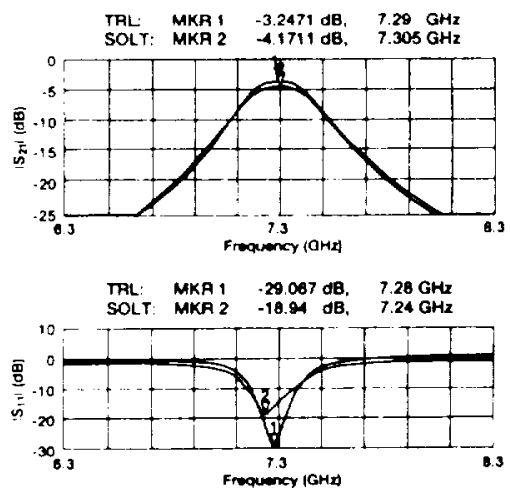

Figure 13. Insertion $\left(S_{21}\right)$ and return $\left(S_{11}\right)$ losses vs. frequency for a gold two-pole bandpass filter with a $7.3 \mathrm{GHz}$ center frequency. Both calibrations were performed at room temperature as shown in Figure 12.

Table 2. Insertion $\left(S_{21}\right)$ and return $\left(S_{11}\right)$ losses for the TRL and SOLT at various temperatures

\begin{tabular}{|c|c|c|c|c|}
\hline Calibration & & $298 \mathrm{~K}$ & $100 \mathrm{~K}$ & $77 \mathrm{~K}$ \\
\hline TRL & \multirow{2}{*}{$\begin{array}{l}\text { Insertion } \\
\text { Loss }\left(S_{21}\right)\end{array}$} & $-3.2471 \mathrm{~dB}$ & $-2.5173 \mathrm{~dB}$ & $-1.876 \mathrm{~dB}$ \\
\hline SOLT & & $-4.1711 \mathrm{~dB}$ & $-3.1511 \mathrm{~dB}$ & $-2.0143 \mathrm{~dB}$ \\
\hline TRL & \multirow{2}{*}{$\begin{array}{l}\text { Return } \\
\text { Loss }\left(S_{11}\right)\end{array}$} & $-29.067 \mathrm{~dB}$ & $-27.588 \mathrm{~dB}$ & $-18.957 \mathrm{~dB}$ \\
\hline SOLT & & $-18.94 \mathrm{~dB}$ & $-15.543 \mathrm{~dB}$ & $-13.868 \mathrm{~dB}$ \\
\hline
\end{tabular}
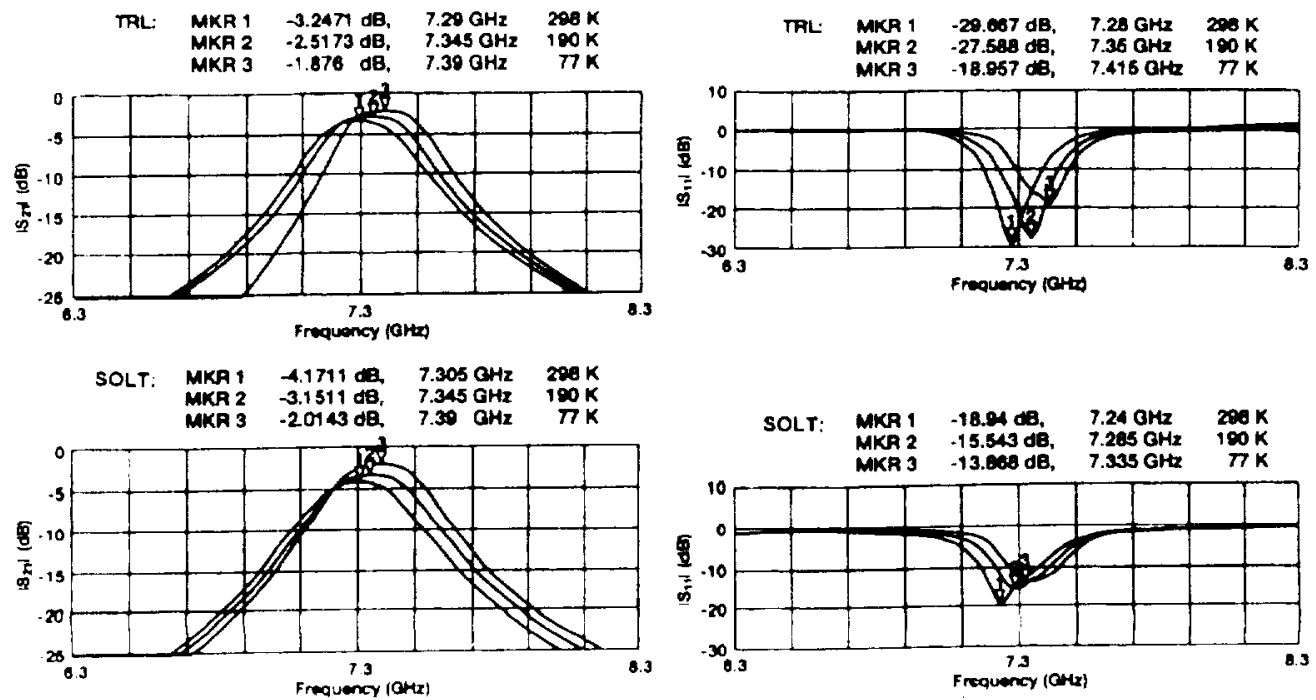

Figure 14. Insertion $\left(S_{2 t}\right)$ and return losses $\left(S_{11}\right)$ vs. frequency for a gold two-pole bandpass filter with a $7.3 \mathrm{GHz}$ center frequency as a function of temperature. 
To begin the initial verification of the TRL calibration at cryogenic temperatures, the test fixture was calibrated at room temperature using both the SOLT and the TRL techniques. The SOLT calibration was performed inside the vacuum can so that the reference planes were established at the plug (male) end of the SMA launchers. The TRL calibration was performed outside the vacuum chamber outside the vacuum chamber at the end of the coaxial cables (See Figure 12). For this test, a different filter of the same aforementioned type was used. Figure 13 shows room temperature results for the filter using the TRL and the SOLT calibrations. As for the previous case, the quality of the insertion and return losses measured using the TRL fixture is better than that obtained using the SOLT calibration. This remains true when comparing the data measured after calibrating at several cryogenic temperatures using the TRL technique with data obtained at the same cryogenic temperatures using the room temperature SOLT calibration (see Figure 14 and Table 2). These results demonstrate that the single-block TRL calibration technique can be effectively used for the fast and reliable characterization of microwave components at room temperature, as well as at cryogenic temperatures. Further improvements of this fixture, such as modifications to allow for the characterization of DUTs of different sizes, and the integration of solid state switches outside the vacuum chamber that would allow for the electronic switching of the standards without altering coaxial connections between the ANA and the TRL setup, are under way. This should further enhance the calibration and measurement speed, as well as the electromagnetic coupling repeatability.

\section{CONCLUSIONS}

A single-block TRL test fixture for the cryogenic characterization of planar microwave components has been designed. fabricated, and tested. The performance for this fixture was superior to that of a commonly used SOLT calibration, both at room and at cryogenic temperatures. In addition, this novel TRL fixture eliminates the repeated temperature cycling and vacuum disruption unavoidable in the performance of calibrations at cryogenic temperatures using other techniques such as the "split-block" TRL configuration. This results in a faster, more accurate, and more affordable calibration and measurement process for the evaluation of the intrinsic properties of individual microwave components. Modifications to allow for the characterization of devices of different sizes and the performance of electronic switching are being considered.

\section{REFERENCES}

1. M.W. Pospiesralski, S. Weinreh, R.D. Norrod, and R. Harris, FET's and HEMT's at cryogenic temperatures-their properties and use in low-noise amplifiers, IEEL Trans. Mirrowave Theory Tech. 36: 552-560 (1988).

2. F.A. Miranda, S.S. Toncich, and K.B. Bhasin, Performance of two-pole handpass fillers photodefined on double-sided Y-Ba-(u-() and Tl-Ba-Ca-Cu-() thin films, Mirrewase and optical Tech. Lett. 6: $752-755(1993)$.

3. J.W. Smuk, M.G. Stuhbs, J.S. Wight, S-Parameter characterization and modeling of three-terminal semiconductive devices at cryogenic temperatures, IELE Microwave and Giaded Wave Letters 2:111-113 (1992).

4. Product Note HP-8510-5A, Network Analysis: Specilying Calibration Standards tor the HP 8510 network analyzer (1991).

5. em ${ }^{6}$. Relualse 2.4. Sonnel Software, Inc. Liverpool NY (1993). 